\title{
BMJ Open Burden and impact of chronic cough in UK primary care: a dataset analysis
}

\author{
James H Hull (D) , ${ }^{1}$ Haya Langerman, ${ }^{2}$ Zia Ul-Haq, ${ }^{3}$ Tahereh Kamalati, ${ }^{3}$ \\ Amanda Lucas, ${ }^{3}$ Mark L Levy (1) ${ }^{4}$
}

To cite: Hull JH, Langerman $\mathrm{H}$ Ul-Haq Z, et al. Burden and impact of chronic cough in UK primary care: a dataset analysis. BMJ Open 2021;11:e054832. doi:10.1136/ bmjopen-2021-054832

- Prepublication history and additional supplemental material for this paper are available online. To view these files, please visit the journal online (http://dx.doi.org/10.1136/ bmjopen-2021-054832).

Received 11 August 2021 Accepted 25 November 2021

Check for updates

(c) Author(s) (or their employer(s)) 2021. Re-use permitted under CC BY-NC. No commercial re-use. See rights and permissions. Published by BMJ.

${ }^{1}$ Department of Respiratory Medicine, Royal Brompton and Harefield NHS Foundation Trust, London, UK

${ }^{2} \mathrm{MSD}$, London, UK ${ }^{3}$ Imperial College Health Partners, London, UK

${ }^{4}$ Self Employed General Practitioner, London, UK

Correspondence to

Dr Mark L Levy;

mark-levy@btconnect.com

\section{ABSTRACT}

Objective Chronic cough (CC) is a debilitating respiratory symptom, now increasingly recognised as a discrete disease entity. This study evaluated the burden of $\mathrm{CC}$ in a primary care setting.

Design Cross-sectional, retrospective cohort study. Setting Discover dataset from North West London, which links coded data from primary and secondary care. The index date depicted CC persisting for $\geq 8$ weeks and was taken as a surrogate for date of $\mathrm{CC}$ diagnosis.

Participants Data were extracted for individuals aged $\geq 18$ years with a cough persisting $\geq 8$ weeks or cough remedy prescription, between Jan 2015 and Sep 2019.

Main outcome measures Demographic characteristics, comorbidities and service utilisation cost, including investigations performed and treatments prescribed were determined.

Results CC was identified in 43453 patients from a total cohort of $2109430(2 \%)$. Median (IQR) age was 64 years (41-87). Among the cohort, $31 \%$ had no recorded comorbidities, $26 \%$ had been given a diagnosis of asthma, $17 \%$ chronic obstructive pulmonary disease, $12 \%$ rhinitis and $15 \%$ reflux. Prevalence of CC was greater in women $(57 \%)$ and highest in the $65-74$ year age range. There was an increase in the number of all investigations performed in the 12 months before and after the index date of CC diagnosis, and in particular for primary care chest $X$-ray and spirometry which increased from 6535 to 12880 and from 5791 to 8720 , respectively. This was accompanied by an increase in CC-associated healthcare utilisation costs. Conclusion One-third of individuals had CC in the absence of associated comorbidities, highlighting the importance of recognising $\mathrm{CC}$ as a condition in its own right. Overall outpatient costs increased in the year after the CC index date for all comorbidities, but varied significantly with age. Linked primary-care datasets may enable earlier detection of individuals with CC for specialist clinic referral and targeted treatment.

\section{INTRODUCTION}

Chronic cough (CC), defined as a cough with a duration of $\geq 8$ weeks in adults, is a debilitating and pervasive symptom with a deleterious impact on quality of life. ${ }^{12}$ Historically, CC has been considered to be and assessed almost exclusively as the component of a potential serious disease process, such as thoracic malignancy, or as part of a symptom complex arising from an undertreated or
Strengths and limitations of this study

This study provided a whole system view of the patient journey throughout their medical care, and enabled a direct assessment of chronic cough on healthcare utilisation and quantification of the associated economic cost.

- It also estimated annual costs of treating chronic cough alone and with comorbid conditions.

- The data provide an important snapshot of how chronic cough is managed, without formal diagnosis, in current UK general practice.

- The study may have been limited by the quality of routinely entered data, which is dependent on accurate coding by clinicians.

unrecognised underlying respiratory disease, such as asthma or chronic obstructive pulmonary disease (COPD), gastro-oesophageal reflux disease (GORD) or nasal disease. ${ }^{3}$ These algorithms were subsequently updated in a later study, which confirmed the concepts for diagnosing CC were still appropriate. ${ }^{4}$ More recently, however, CC is becoming increasingly recognised to exist as a discrete 'disease' entity. ${ }^{5}$ Potential underlying pathologies may involve disordered neural sensitivity, ${ }^{67}$ a disbalance of peripheral activation, or altered central cough control. ${ }^{8}$

Accordingly, it is recognised that individuals with CC frequently describe neurogenic features such as hypertussia and alotussia, and these features are akin to symptoms described in other neural hypersensitivity disorders such as diabetic neuropathy. ${ }^{9}$ Indeed, this improved recognition of CC as a hypersensitive or neural-based process has driven development of targetted non-pharmacological (eg, speech and language therapy targeting cough suppression) and pharmacological therapies (eg, P2X3 inhibition ${ }^{10}{ }^{11}$ ) now in various stages of development. ${ }^{12}$

To date, there are only limited data available describing the epidemiology, characteristics and comorbid factors of individuals with CC in the general population. A 2015 systematic review and meta-analysis to estimate the 
epidemiological burden of CC in general adult populations reported a high global prevalence of $9.6 \% .^{13}$ However, the authors acknowledged that more than $80 \%$ of the included studies were not primarily focused on CC and 19 different definitions were used. The reported prevalence rates for CC in the UK vary widely. In the UK, a questionnaire-based survey by Cullinan, with clinical investigations performed in a sample of those interviewed, reported that $14.1 \%$ of men and $10.1 \%$ of women reported a cough that affected them every day, or for at least 6 months. ${ }^{14}$ In 2006, a primary care-based study estimated CC prevalence to be approximately $12 \%$, based on a random sample of individuals from 36 UK general practices. ${ }^{15}$ More recently, a retrospective cohort study conducted using primary care data from the UK Clinical Practice Research Datalink (CPRD GOLD), which included records for over 198000 individuals with codingbased CC identification criteria, reported a much lower annual prevalence of $0.2 \%$ for probable CC and $1.2 \%$ for possible CC. ${ }^{16}$ There thus remains a degree of disparity and uncertainty regarding the community frequency of CC and other characteristics, such as demographics, referral patterns and cost of treatment.

Discover consists of a longitudinal North West London (NWL) dataset, covering linked coded primary care, secondary care, acute, mental health, community health and social care records for over 2.5 million individuals who live and are registered with a General Practitioner (GP) in NWL. It is fed by data from over 400 provider organisations including 360 GP practices, 2 mental health and 2 community trusts and all acute providers attended by NWL inhabitants. ${ }^{17}$ The aim of this crosssectional primary-care based evaluation study was to use this comprehensive dataset to explore and describe the demographic characteristics, comorbidities and assessment pathway of a NWL CC cohort, described by age categories, and to evaluate cost associated with this condition.

\section{METHODOLOGY}

\section{Data source: Discover dataset}

This cross-sectional, retrospective cohort study extracted data from the Discover dataset, which uses the Whole System Integrated Care database in a deidentified form, and is geographically located in the NWL region. The coded data from primary and secondary care are linked to create a single, integrated record for each individual. It covers prescriptions and events including inpatient, outpatient, Accident and Emergency/Emergency Department (A\&E/ED) activity, diagnosis and investigation codes. The primary care dataset contains data on patient demographics, medical history, consultations, referrals, prescriptions and test results.

\section{Patient and public involvement}

This was a deidentified dataset analysis and as such there was no direct patient involvement.

\section{Study subjects}

Over the period 1 January 2015-30 September 2019, adults $\geq 18$ years of age in the Discover dataset with CC were identified. Patients with CC were defined as those with two or more recorded consultations coded as 'cough-related' persisting for at least 8 weeks, and this definition was unchanged throughout the study. Primary and secondary care data became linked to the Discover dataset from 2015, hence the choice to use 2015-2019 for the study period. The point in time/date (visit 2, the index date) was defined as a surrogate for the date of CC diagnosis, when the subject first met the criterion for $\mathrm{CC}$ (ie, persisting for at least 8 weeks).

\section{Study design}

One hundred and ninety primary care data entry codes for cough (online supplemental document 1) and secondary care ICD-10 code entries, and/or a prescription for a remedy that contained a component that is only used in the treatment of cough were used to identify the CC cohort. At least one of the cough coded entries had to be present at both the first and second visits. To characterise the interaction with comorbidities and to determine the CC cases likely to lead to higher NHS service utilisation, data on four associated GP-diagnosed comorbidities (asthma, COPD, rhinitis and reflux), smoking status, ACE inhibitor prescriptions, investigations and outpatient costs were extracted for a 12-month period prior to the index date and a 12-month period after the index date (after CC diagnosis) (figure 1). Treatments presumed to have been administered for CC (eg, cough suppressants, demulcents and expectorants) and antibiotics for respiratory tract infections were identified and their prescription before and after the index date was described.

Cost of CC was evaluated by determining the primary care and outpatient appointments and investigations (chest X-ray, CT scan, spirometry) at 2019 cost tariff. Costs did not include prescription or inpatient care associated costs. For details on cost calculations, refer to online supplemental document 2. Inpatient episodes and costs were excluded because the Discover dataset only has access to the final International Classification of Diseases, Tenth Revision (ICD-10) coded discharge diagnosis and not whether CC was the reason for the admission.

\section{Statistical analyses}

Data were reported using descriptive statistics as median (IQR) unless otherwise stated. No further detailed statistical analyses were carried out. The costs of treating individuals with $\mathrm{CC}$, and mean per patient and total costs of treating individuals with $\mathrm{CC}$, by year, were derived.

\section{RESULTS}

\section{Prevalence and characteristics of CC}

Data were successfully extracted from 350 GP practices, covering a total of 2109430 individuals, and identifying a cohort of 43453 from 2109430 (2\%) adults >18 years 
Chronic cough

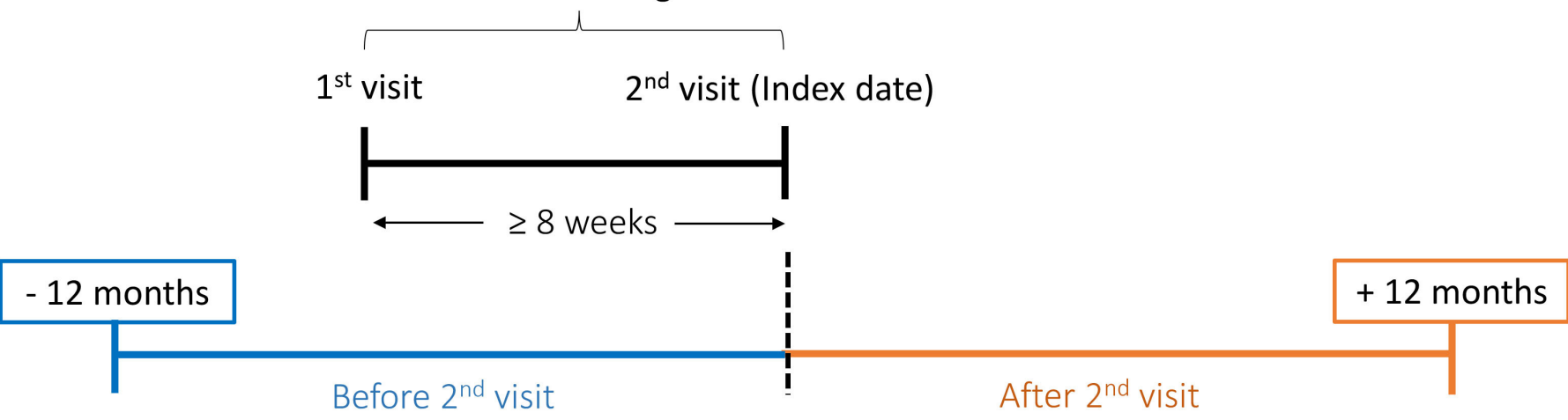

Figure 1 Illustration of point at '2nd Visit' where chronic cough was defined in the study. Chronic cough was diagnosed once there were two or more recorded consultations coded as 'cough-related' persisting for at least 8 weeks. At least one of the cough coded entries had to be present at both the first and second visits.

(24 $565(57 \%)$ women) with CC. The median (IQR) age of individuals was 64 years (41-87) and the 65-74year age range had the highest proportion of individuals with $\mathrm{CC}$ within the total CC cohort (table 1, figure 2); only 6755 $(15.5 \%)$ individuals were aged under 40 years old.

A total of $6335(14.6 \%)$ individuals were recorded as being prescribed an ACE inhibitor before the index date. Smoking status was only retrievable for $44.2 \%$ of the CC cohort; however, overall $22.3 \%$ of indiviuals with CC were current smokers, $18.8 \%$ previous smokers and $3.1 \%$ nonsmokers. Of the CC cohort, $34.3 \%$ were classified by body mass index (BMI) as overweight (BMI $\geq 25$ to $\left.<30 \mathrm{~kg} / \mathrm{m}^{2}\right)$, $27.3 \%$ were obese $\left(\mathrm{BMI} \geq 30\right.$ to $<40 \mathrm{~kg} / \mathrm{m}^{2}$ ), and $4.4 \%$ severely obese $\left(\mathrm{BMI} \geq 40 \mathrm{~kg} / \mathrm{m}^{2}\right)$.

\section{CC-associated comorbidities}

At least one CC-associated comorbid condition was noted in $30102(68 \%)$ individuals (table 1). During the study period, a diagnosis of asthma was coded in the records of 11435 (26\%), COPD in 7509 (17\%), reflux in 6354 (15\%) and rhinitis in $5111(12 \%)$ (table 1). The proportion of individuals with one of these comorbidities increased gradually with age from around $50 \%$ at age $18-24$ years to around $75 \%$ at age $65-69$ years (figure 3 ). The exception was rhinitis, where the proportion was highest in younger individuals ( $<45$ years) and then started to decline in middle age and thereafter (table 1, figure 3). Of these comorbidities, all were greater in current smokers or previous smokers compared with non-smokers, and previous smokers had only slightly fewer comorbidities than current smokers.

\section{Time from first GP appointment for CC to first referral for an outpatient appointment and time to outpatient appointment and investigations}

The median (IQR) delay from the index date of diagnosed CC to a referral for an outpatient appointment was 4.0 (1.0-6.0) months for respiratory medicine, 5.0 (1.0-6.0) months for Ear Nose and Throat Disorders (ENT), and 5.0 (2.0-5.0) months for gastroenterology, with an overall median delay of 4.0 (1.0-6.0) months. The median (IQR) delays between index date and first outpatient appointment were 6.0 (3.0-9.0), 7.0 (4.0-9.0) and 7.0 (4.0-9.0) months, for respiratory medicine, ENT and gastroenterology, respectively, with an overall median delay of 6 (4.0-9.0) months.

In the CC cohort, there was an increase in the number of all investigations performed in the 12 months after the $\mathrm{CC}$ index date. Increases in primary care chest X-ray (from 6535 to $12880,49 \%$ increase), and primary care spirometry (from 5791 to $8720,34 \%$ increase) were observed in the year after a CC diagnosis. In the CC cohort, outpatient spirometry investigations rose from a total of 486 investigations in the year before a CC diagnosis to 1093 in the year after, an increase of $56 \%$.

A delay of 8 (3-18) months was noted between the CC index date and day of first spirometry carried out in primary care; the delay between the CC index date and first secondary care spirometry was even longer at 17 (8-31) months.

\section{CC-associated healthcare utilisation costs}

For all comorbidities, outpatient costs per patient per year (ppy) for treatments prescribed and investigations were greater after the CC index date than before. Coughassociated outpatient ppy costs were highest for reflux. CC with comorbid asthma and COPD had the second highest costs ppy, followed by smoking and rhinitis (figure 4, online supplemental tables 1-5). Outpatient costs were also greater after the CC index date in those with CC without any comorbidities; however, these costs were lower in those without than in those with CC and comorbid conditions (figure 4).

Costs ppy increased with age both in the 12 months before and 12 months after the CC index date, but for each age category the costs were again considerably greater in the year after the index date than before.

Outpatient costs were also examined in smokers before and after the CC index date. The highest increase in costs $(39 \%)$ due to cough was in the 50-54year age group; an increase of $£ 223$ ppy. 


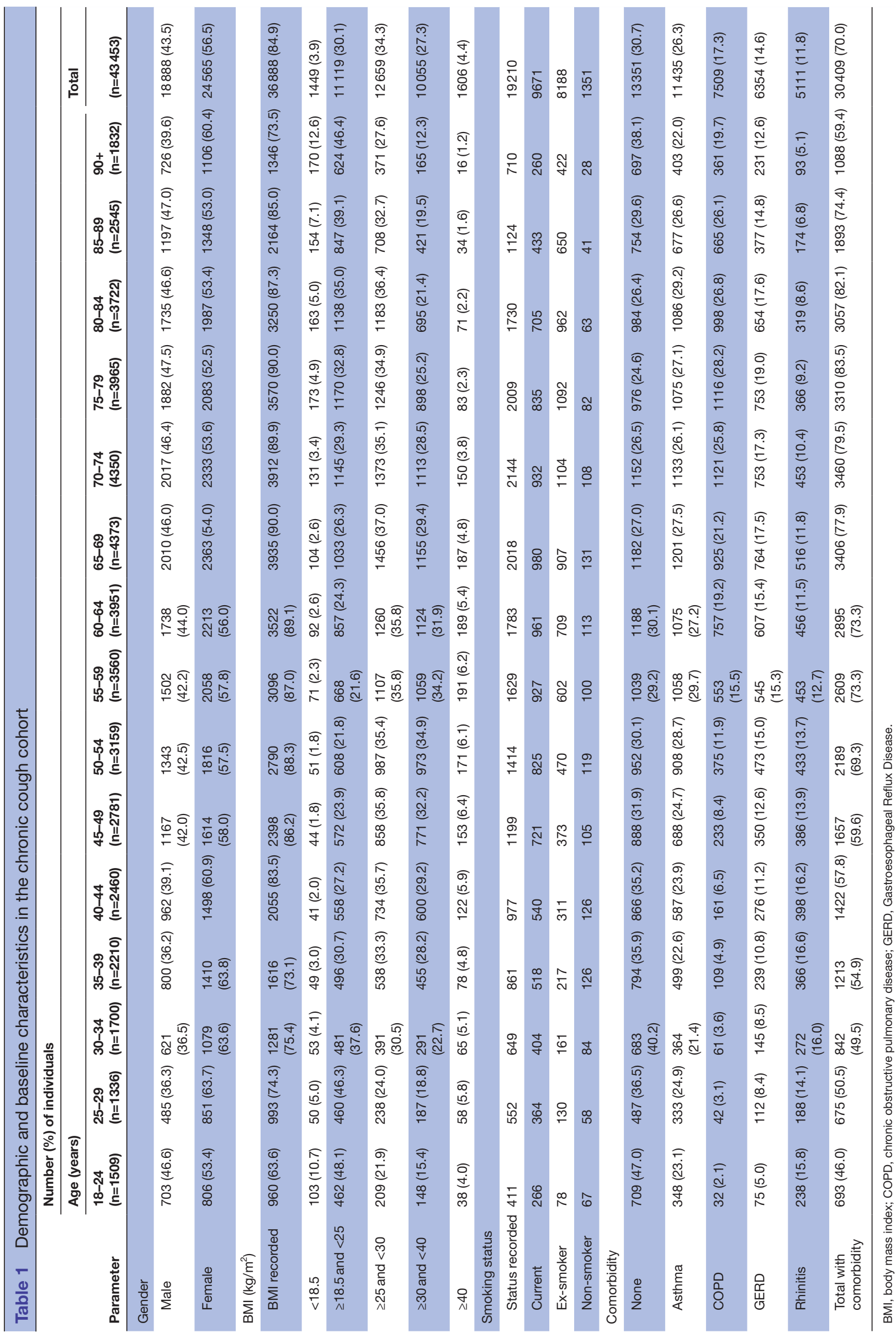




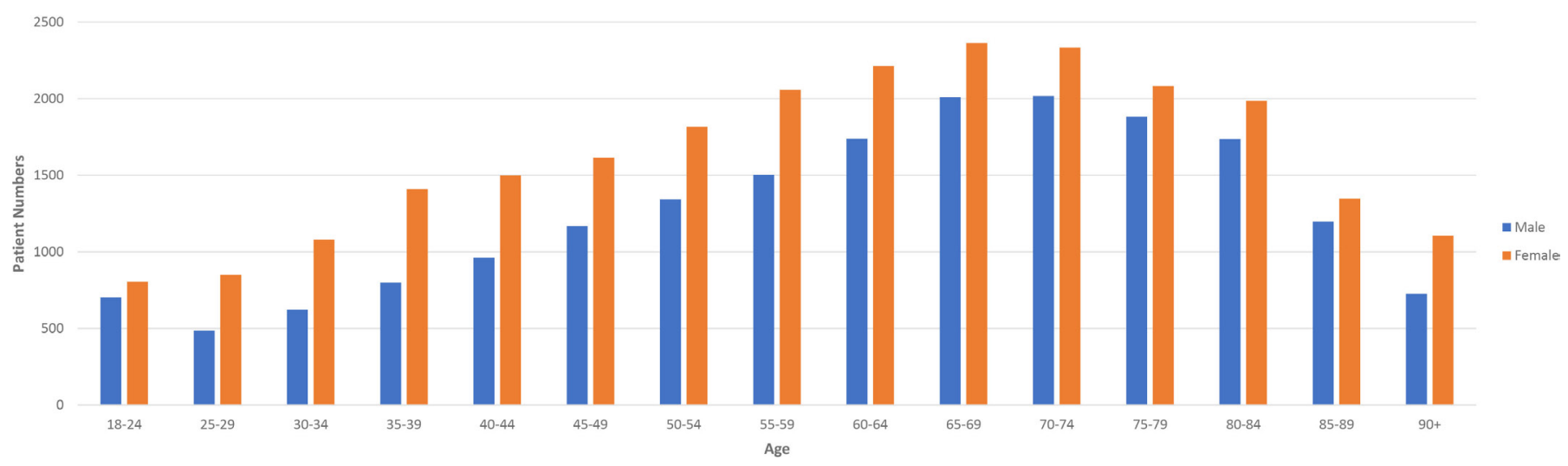

Figure 2 Chronic cough cohort by age and gender. Median (IQR) age was 65 (49-77) years for men and 62 (46-75) years for women.

\section{Treatments}

At least one prescription for an antibiotic was issued for 24592 (56.6\%) individuals in the CC cohort over the study period. However, nearly two-thirds of the CC cohort (27 $501(63 \%))$ had not been prescribed any cough remedy. The most commonly prescribed classes of antibiotic were from the penicillin class, which comprised $44.3 \%$ of antibiotic prescriptions. A prescription for morphine that might have been used to treat cough was issued for 601 $(1.4 \%)$ individuals.

\section{DISCUSSION}

In this large UK primary care dataset study, CC was present in approximately $2 \%$ of adults, and most frequently reported in women and in the 65-74year age range. These findings provide further insight regarding the epidemiology of CC in the community and broadly align with the characteristics of patients attending specialist cough clinics in the $\mathrm{UK}^{18}$; that is, indicating a preponderance of CG in later-middle aged women. ${ }^{19}$

In the assessment of CC, several causative or potentially related clinical issues are commonly considered in the diagnostic and therapeutic workup. Accordingly, we found that two-thirds of individuals with CC in this study were listed as having comorbid conditions on their medical record; the most common being asthma
(26.3\%), followed by COPD (17.3\%), reflux (14.6\%) and rhinitis $(11.8 \%)$. We also found ACE inhibitor prescription reported in approximately $15 \%$ and this is clearly a potentially relevant factor in precipitating CC in some. ${ }^{20}$ These findings align with prior published work in this field $^{162122}$ and reaffirm the challenge of deciphering the relationship between $\mathrm{CC}$ arising as a manifestation of undertreated disease versus coexisting cough hypersensitivity or indeed misdiagnosis. Regardless, the subgroup of individuals with CC without any listed comorbidities, substantially comprised a third of the current study population (ie, CC arising alone represented $0.6 \%$ of overall population), supporting the assertion that CC appears to arise in the absence of coexisting symptoms driving an alternative diagnosis. Potential mechanisms include disordered neural sensitivity, ${ }^{67}$ as well as an imbalance of peripheral activation and central control of cough. ${ }^{8}$ This may occur because of increased exposure of the sensory nerve terminals to inflammatory mediators, chemical irritants or excess mucus, or because of changes in the excitability of neuronal pathways that may affect airway sensory nerves and/or their central nervous system connections. ${ }^{8}$

The most comparable dataset to this study is that of the recently published CPRD-GOLD study. ${ }^{16}$ Holden et al evaluated 150231 adults using a broader representation of a UK-based primary care population, but also reporting

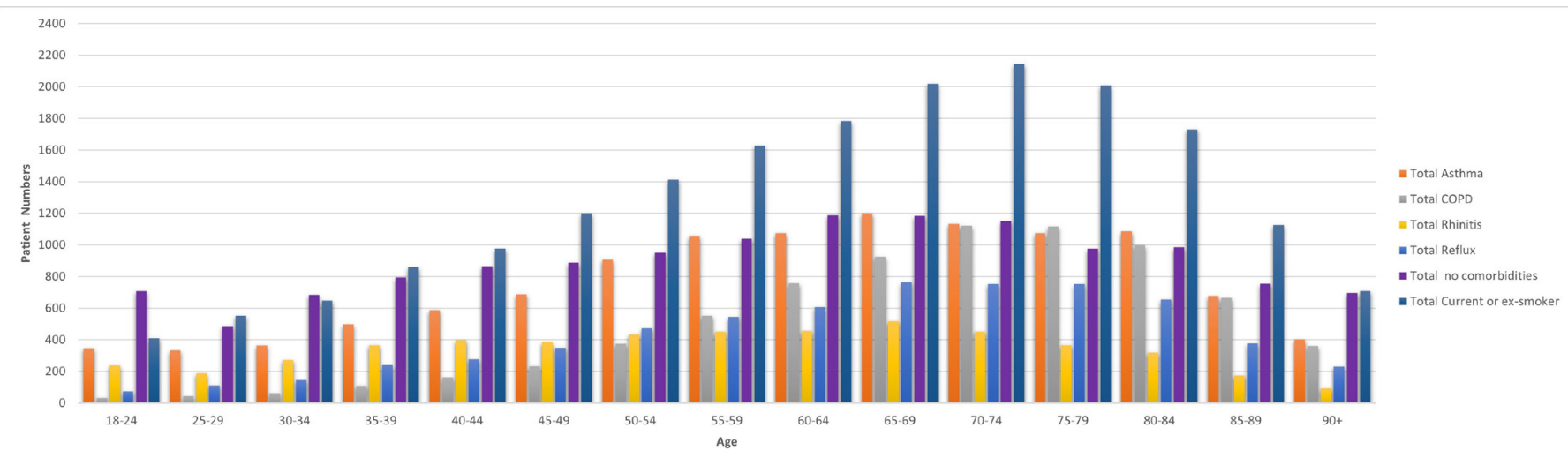

Figure 3 Chronic cough cohort by age and comorbidities. 


\section{Annual costs within co-morbidity cohorts before and after Chronic Cough Index Date}

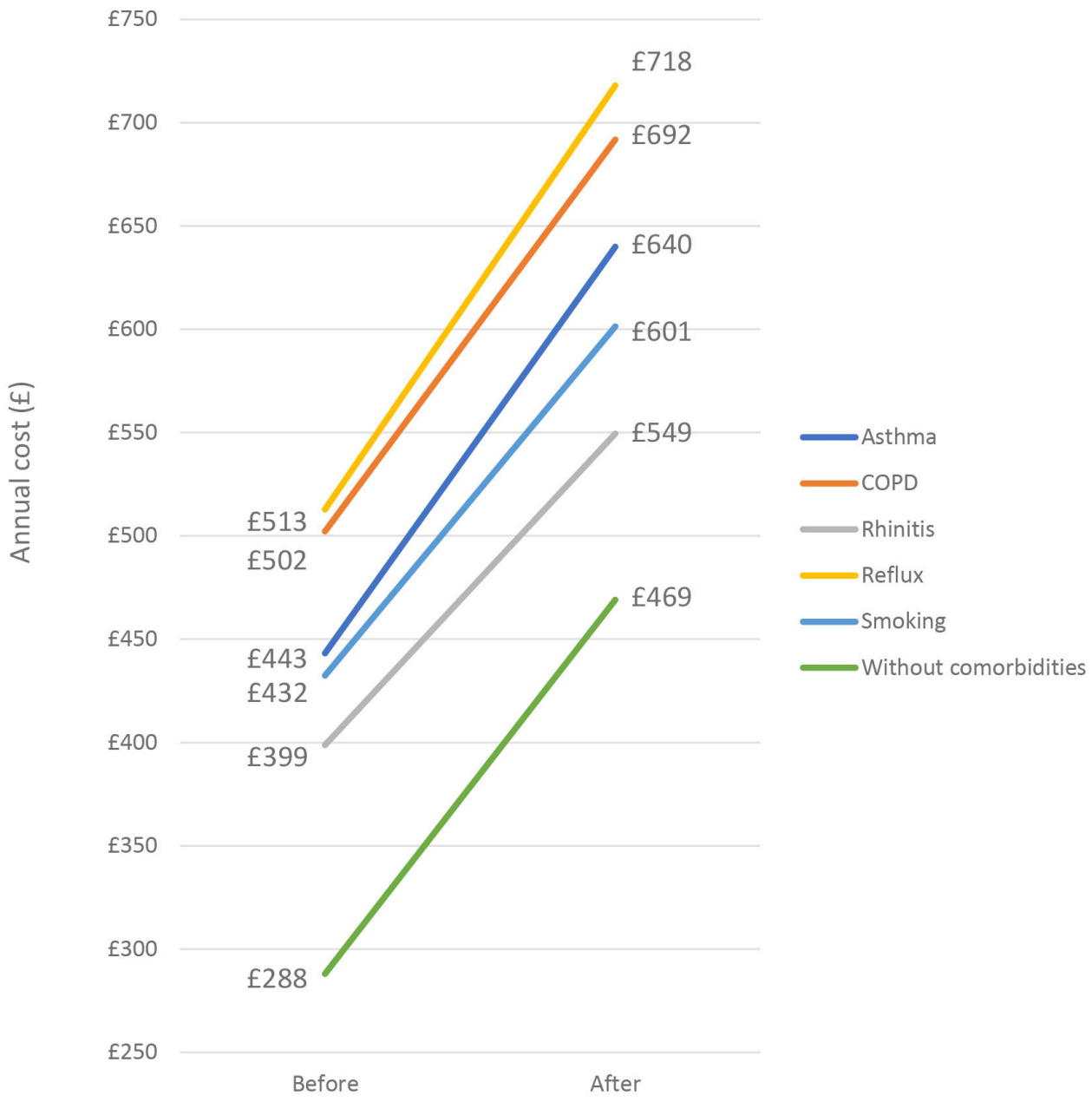

Figure 4 Costs per patient per year in those subjects with chronic cough without and with comorbidities before and after chronic cough index date. COPD, chronic obstructive pulmonary disease.

acute cough events. They found a lower prevalence estimate of probable CC $(0.18 \%)$, but a roughly similar prevalence using an overall process-specific definition (1.2\%). The patient demographic and baseline characteristics were also similar to the current study in terms of age (61 and 67 years in probable and possible CC groups, respectively, vs 64 years in current study), proportion of women (56\% and $61 \%$ in probable and possible CC groups, respectively, vs $57 \%$ ), and ACE inhibitor exposure $(11.5 \%$ vs $14.6 \%)$. It is unclear why the prevalence of CC reported in both of these UK-based primary care dataset studies differs in comparison with the questionnaire study by Cullinan in which $14.1 \%$ of men and $10.1 \%$ of women reported CC, ${ }^{14}$ but a potential explanation relates to the CC definitions and age inclusion criteria used.

As in the study by Holden $e t a l^{16}$ we found a high proportion (one in five) of individuals with CC were either current or ex-smokers. In comparison, UK national data indicate that in 2019 the proportion of current smokers aged $\geq 18$ years was $14.1 \%$. ${ }^{23}$ Smoking is a risk factor for diverse respiratory as well as other diseases, and comorbid conditions, and has been shown to adversely affect treatment efficacy in previous studies of CC. ${ }^{24}{ }^{25}$ It is also a relevant factor in promoting attendance for primary care review. Our findings also align with respect to the finding that individuals with CC are frequently referred to a wide range of secondary care specialists and undergo multiple investigations. ${ }^{26}$ Whether CC-associated costs are increased by the presence of comorbid conditions could not be determined in this study. However, repeated trials of treatment and diagnostics in people suffering from ongoing symptoms of CC are likely to contribute to increased outpatient costs. For each comorbidity, outpatient costs were greater after the CC index date than before. Costs peaked at around 70-85 years of age and were higher for individuals with COPD or GORD than rhinitis.

Over half the CC cohort (56.6\%) was issued at least one prescription for an antibiotic. Again this broadly aligns with other studies evaluating pathways of care for CC in primary care ${ }^{16}$ The prescription of antibiotics where there is uncertainty over whether a bacterial infection is 
present is costly and can lead to antibiotic resistance. This reaffirms a need for improved access to diagnostic investigational pathways that can confirm or refute the presence of bacterial infection, as a key component of improved antibiotic stewardship. The proportion receiving prescriptions for cough remedies was relatively low, but this is not unexpected as it is customary for individuals in the UK to be directed to buy these over the counter.

We have demonstrated increased outpatient costs following the index date for CC diagnosis in individuals with a range of common CC-associated comorbidities. There is also evidence that the presence of comorbid CC indicates a more severe form of the comorbid disease. A comparison of individuals with asthma with and without CC among 14740 adults from the Copenhagen General Population Study found that those with CC suffered from more severe respiratory symptoms, greater healthcare utilisation, lower lung function and higher levels of systemic inflammatory biomarkers. ${ }^{27}$ Using the same database, comorbid CC was also associated in individuals with COPD, with more respiratory symptoms and healthcare utilisation ( $\geq 3$ outpatient visits during the past 12 months), lower lung function and increased inflammation. ${ }^{28}$

It was not possible to ascertain whether individuals with a comorbidity incurred increased costs after the index date due to chronic refractory cough unrelated to the comorbid condition, ${ }^{5}$ or whether the comorbidity was undertreated in this population. Similar findings have been reported in studies evaluating the economic costs of asthma, which reported that those subjects with severe or poorly controlled asthma were responsible for a large proportion of the costs. ${ }^{29} 30$

\section{Strengths and limitations}

The dataset used in this study provided a whole system view of the patient journey throughout their medical care, and enabled a direct assessment of CC on healthcare utilisation and quantification of the associated economic cost (via access to accurate commissioner's costs at patient level). It also provided an estimate of annual costs of treating $\mathrm{CC}$ alone and with comorbid conditions.

This study, as in the case of other dataset studies, was limited by the quality of routinely entered data which is dependent on accurate coding by clinicians. General practitioner's may use free text entries which cannot be extracted into the dataset, or a variety of symptomdriven coded data entry, with or without the use of standardised templates, and furthermore, they may not use specific diagnostic codes (see online supplemental document 1 ). We acknowledge that the nature of the analysis undertaken did not permit interrogation of the validity or robustness of a listed diagnosis and thus although 'doctor diagnosed asthma' for example may be listed as a 'co-morbidity', the basis for this diagnosis may not have been underpinned by objective confirmatory tests, and in some cases may have been based on the presence of CC. Some comorbidities are hard to detect and and it can be difficult to determine which came first, the comorbidity or the CC. The primary care records, which are contemporaneous, provide the ability to determine which came first, but these were not included in the current analysis. A further limitation of this study is that the frequency of cough codes between the first and second visits was not collected. The number of individuals excluded from the study due to missing coding is not quantifiable. Individuals with repeated acute cough episodes may be classified using data entry codes as having CC instead of recurrent cough in error or vice versa. A patient and/or a general practice may also 'opt out' or 'dissent' from sharing data across any care setting, meaning that no data will appear for those individuals in the extracted data.

The Discover dataset covers a diverse NWL population, but may not be generalisable to the rest of the UK. Resource utilisation presented in this study reflects United Kingdom National Health Service (NHS) resources as determined by the commissioner's referral policies and pricing and does not include the true cost of provision of primary care services, the cost to individuals or private healthcare costs and therefore the entire economic burden of the disease is not completely described in this research. Nevertheless, the data provide an important snapshot of how CC is managed, without formal diagnosis, in current UK general practice. For the two-thirds of individuals in this cohort presenting with one or more CC-associated comorbidities, efforts are required to determine whether the CC is a manifestation of undertreatment of the comorbid condition and/or due to hypersensitivity. Analysis of the extent to which chronic comorbidities were optimally treated was beyond the scope of this study, but is an important topic for future research.

\section{Conclusion}

In this cross-sectional primary-care based evaluation, CC was present in $2 \%$ of adults, contributing to considerable morbidity and health economic burden. One-third of individuals had ongoing CC symptoms in the absence of associated comorbidities. It is unclear whether the CC in the other two thirds was due entirely to their diseases or exaggerated by underlying hypersensitive cough. In line with the latest European Respiratory Society guidelines on the diagnosis and treatment of $\mathrm{CC},{ }^{5}$ efforts should be directed towards recognition of $\mathrm{CC}$ as a condition in its own right with different phenotypes (asthmatic/ eosinophilic cough, reflux cough, upper airways cough syndrome and iatrogenic cough). Management should be based on a combination approach of selected diagnostic testing and empirical trials of treatment. Resource is required to better characterise optimal intervention strategies to improve care and for appropriate allocation of resources for individuals including specialist clinic referral and targeted treatment.

Twitter Mark L Levy @bigcatdoc

Contributors MLL contributed to development of the protocol, provided expertise on general practice and the Discover dataset, was involved with decisions related 
to data extraction and analysis, and with writing and co-editing the paper. $\mathrm{AL}$ contributed to development of the protocol, provided expertise related to the Discover dataset, and was involved with decisions related to data extraction and analysis. AL is also the guarantor for the data. $\mathrm{ZU}-\mathrm{H}$ contributed to development of the protocol, provided expertise related to the Discover dataset, was involved with decisions related to data extraction and analysis, performed all the data extraction, analysis and visualisation. TK contributed to development of the protocol, provided expertise related to the Discover dataset, was involved with decisions related to data extraction, analysis and visualisation. $\mathrm{JH}, \mathrm{MLL}$ and $\mathrm{HL}$ conceptualised and designed this work, reviewed results and analysis and created and coauthored the manuscript. Editorial assistance was provided by Jenny Grice, a freelance medical writer.

Funding This study was funded by MSD, a subsidiary of Merck \& Co., Inc., Kenilworth, NJ, USA: award/grant number N/A. The funders played a role in study design, interpretation of results, decision to publish and preparation of the manuscript.

Competing interests $\mathrm{JH}$ has received payment for advisory board and consultancy work with MSD Pharmaceuticals and Bellus Health Inc. MLL Reports: Fees for: Consultancy - Clement Clarke International, Boehringer Ingelheim, AstraZeneca, GSK, Orion, TEVA pharmaceuticals, Menarini, NSHI, Chiesi Pharmaceuticals, Novartis, Discover-Now. Fees for: Lectures - TEVA, Novartis, Orion, AstraZeneca, NAPP, Chiesi, NSHI. GINA (Travel expenses and accommodation only) Honoraria: ADMIT Group - Consorzio Richerche Ferrara.

Patient consent for publication Not applicable.

Ethics approval Discover data are deidentified to meet data minimisation standards of the Information Standards Boards of NHS Digital. Ethics approval for the use of the deidentified dataset is currently provided by the Information Governance (IG) Board. Ethical approval for the use of the Discover Platform for research was obtained (REC reference is 18/WM/0323 and the IRAS project ID is 253449).

Provenance and peer review Not commissioned; externally peer reviewed.

Data availability statement Data may be obtained from a third party and are not publicly available. The Discover dataset depository is not available for public access.

Supplemental material This content has been supplied by the author(s). It has not been vetted by BMJ Publishing Group Limited (BMJ) and may not have been peer-reviewed. Any opinions or recommendations discussed are solely those of the author(s) and are not endorsed by BMJ. BMJ disclaims all liability and responsibility arising from any reliance placed on the content. Where the content includes any translated material, BMJ does not warrant the accuracy and reliability of the translations (including but not limited to local regulations, clinical guidelines, terminology, drug names and drug dosages), and is not responsible for any error and/or omissions arising from translation and adaptation or otherwise.

Open access This is an open access article distributed in accordance with the Creative Commons Attribution Non Commercial (CC BY-NC 4.0) license, which permits others to distribute, remix, adapt, build upon this work non-commercially, and license their derivative works on different terms, provided the original work is properly cited, appropriate credit is given, any changes made indicated, and the use is non-commercial. See: http://creativecommons.org/licenses/by-nc/4.0/.

\section{ORCID iDs}

James H Hull http://orcid.org/0000-0003-4697-1526

Mark L Levy http://orcid.org/0000-0002-1807-3246

\section{REFERENCES}

1 French CL, Irwin RS, Curley FJ, et al. Impact of chronic cough on quality of life. Arch Intern Med 1998;158:1657-61.

2 Braido F, Baiardini I, Tarantini F, et al. Chronic cough and QoL in allergic and respiratory diseases measured by a new specific validated tool-CCIQ. J Investig Allergol Clin Immunol 2006;16:110-6.

3 Irwin RS, Baumann MH, Bolser DC, et al. Diagnosis and management of cough executive summary: ACCP evidence-based clinical practice guidelines. Chest 2006;129:1S-23.

4 Irwin RS, French CL, Chang AB, et al. Classification of cough as a symptom in adults and management algorithms: CHEST guideline and expert panel report. Chest 2018;153:196-209.
5 Morice AH, Millqvist E, Bieksiene $\mathrm{K}$, et al. ERS guidelines on the diagnosis and treatment of chronic cough in adults and children. Eur Respir J. In Press 2020;55:1901136.

6 Chung KF, McGarvey L, Mazzone SB. Chronic cough as a neuropathic disorder. Lancet Respir Med 2013;1:414-22.

7 Hull JH, Menon A. Laryngeal hypersensitivity in chronic cough. Pulm Pharmacol Ther 2015;35:111-6.

8 Smith JA, Badri H. Cough: new pharmacology. J Allergy Clin Immunol Pract 2019;7:1731-8.

9 Chung KF, Pavord ID. Prevalence, pathogenesis, and causes of chronic cough. Lancet 2008;371:1364-74.

10 Abdulqawi R, Dockry $\mathrm{R}$, Holt $\mathrm{K}$, et al. P2X3 receptor antagonist (AF-219) in refractory chronic cough: a randomised, double-blind, placebo-controlled phase 2 study. Lancet 2015;385:1198-205.

11 Smith JA, Kitt MM, Morice AH, et al. Gefapixant, a P2X3 receptor antagonist, for the treatment of refractory or unexplained chronic cough: a randomised, double-blind, controlled, parallel-group, phase 2B trial. Lancet Respir Med 2020;8:775-85.

12 Jolley CJ, Birring SS. New drug targets for chronic cough: research you can literally sink your teeth into! Eur Respir J 2017;50:1701571.

13 Song W-J, Chang Y-S, Faruqi S, et al. The global epidemiology of chronic cough in adults: a systematic review and meta-analysis. Eur Respir J 2015;45:1479-81.

14 Cullinan P. Persistent cough and sputum: prevalence and clinical characteristics in South East England. Respir Med 1992;86:143-9.

15 Ford AC, Forman D, Moayyedi P, et al. Cough in the community: a cross sectional survey and the relationship to gastrointestinal symptoms. Thorax 2006;61:975-9.

16 Holden SE, Morice A, Birring SS, et al. Cough presentation in primary care and the identification of chronic cough: a need for diagnostic clarity? Curr Med Res Opin 2020;36:139-50.

17 Bottle A, Cohen C, Lucas A, et al. How an electronic health record became a real-world research resource: comparison between London's whole systems integrated care database and the clinical practice research datalink. BMC Med Inform Decis Mak 2020;20:71.

18 Kelsall A, Decalmer S, McGuinness K, et al. Sex differences and predictors of objective cough frequency in chronic cough. Thorax 2009;64:393-8.

19 Morice AH, Kastelik JA. Cough. 1: chronic cough in adults. Thorax 2003;58:901-7.

20 Dicpinigaitis PV. Angiotensin-converting enzyme inhibitor-induced cough: ACCP evidence-based clinical practice guidelines. Chest 2006;129:169S-73.

21 Ryan NM, Vertigan AE, Birring SS. An update and systematic review on drug therapies for the treatment of refractory chronic cough. Expert Opin Pharmacother 2018;19:687-711.

22 Lätti AM, Pekkanen J, Koskela HO. Defining the risk factors for acute, subacute and chronic cough: a cross-sectional study in a Finnish adult employee population. BMJ Open 2018;8:e022950.

23 Office for National Statistics. Adult smoking habits in the UK, 2019. Available: https://www.ons.gov.uk/peoplepopulation andcommunity/healthandsocialcare/healthandlifeexpectancies/ bulletins/adultsmokinghabitsingreatbritain/2019 [Accessed 6 Aug 2020].

24 Pisinger C, Godtfredsen NS, Jørgensen T. Smoking reduction and cessation reduce chronic cough in a general population: the Inter99 study. Clin Respir J 2008;2:41-6.

25 Rojewski AM, Baldassarri S, Cooperman NA. Comorbidities workgroup of the society for research on nicotine and tobacco (SRNT) treatment network. exploring issues of comorbid conditions in people who smoke. Nicotine Tob Res 2016;18:1684-96.

26 Birring SS, Holden SE, Jenkins-Jones S. Healthcare utilization and cost associated with chronic cough in the United Kingdom: a retrospective observational study. ERS International Congress 2020 Virtual. Abstract 23406.

27 Çolak Y, Afzal S, Lange P, et al. Role and impact of chronic cough in individuals with asthma from the general population. J Allergy Clin Immunol Pract 2019;7:1783-92.

28 Landt E, Çolak Y, Lange P, et al. Chronic cough in individuals with COPD: a population-based cohort study. Chest 2020;157:1446-54.

29 Serra-Batlles J, Plaza V, Morejón E, et al. Costs of asthma according to the degree of severity. Eur Respir J 1998;12:1322-6.

30 Puig-Junoy J, Pascual-Argenté N. Costes socioeconómicos de asma en la Unión Europea, Estados Unidos y Canadá: revisión sistemática [Socioeconomic costs of asthma in the European union, United States and Canada: a systematic review]. Rev Esp Salud Publica 2017;91:e201703025. 